\title{
Icy Dwarf Planets: Colored Popsicles in the Outer Solar System
}

\author{
Noemi Pinilla-Alonso ${ }^{1,2}$ \\ ${ }^{1}$ Florida Space Institute: 12354 Research Parkway, partnership 1 Building, Suite 214 \\ Orlando, 32826-0650 \\ email: npinilla@ucf.edu \\ ${ }^{2}$ Department of Earth and Planetary Sciences, University of Tennessee. \\ 306 EPS Building, 1412 Circle Dr Knoxville TN 37996-1410
}

\begin{abstract}
We update the list of candidates to be considered by the IAU as dwarf planets using the criterium suggested by Tancredi \& Favre (2008). We add here the information collected in the last 10 years (mostly the sizes and albedos by the herschel hey program TNOs Are Cool). We compare the physical characteristics of these candidates with the physical characteristics of the rest of the TNOs. Our goal is to study if there are common physical properties among the candidates that enable the identification of a dwarf planet.
\end{abstract}

Keywords. solar system: general, icy bodies, Kuiper Belt

\section{Introduction}

In 1992 the discovery of $1992 \mathrm{QB}_{1}$ (Jewitt \& Luu, 1992) was the trigger of a race to characterize the newly discovered trans-Neptunian belt. The existence of a swarm of "icy asteroids" similar to Pluto orbiting in the outer Solar System, that would be the origin of the short-period comets, had been largely hypothesized but its detection was also an elusive goal (Leonard, 1930, Edgeworth 1943, Kuiper 1951, Fernandez, 1980). This icy belt was for long considered by the planetary scientists as the icy promised land, the largest reservoir of primordial ices in the Solar System (Schmitt et al. 1998, Cruikshank 2005).

From 1992 to 2005 about 1000 trans-Neptunian objects and Centaurs were discovered and a lot of "first ever" science was published e.g. $1996 \mathrm{TO}_{66}$, first ever detection of the water ice bands in a TNO's spectrum (Brown et al. 1999); $1998 \mathrm{WW}_{31}$, first detection of a binary (Veillet et al. 2002); first estimation of size and albedo from thermal and visible observations, Varuna (Jewitt et al. 2001).

Almost 10 years ago, 2005 was the year of the announcement of another unique discovery: the existence of three large icy objects, (136108) Haumea, (136472) Makemake and (136199) Eris (Brown et al. 2005a, Santos-Sanz et al. 2005), only comparable in size with Pluto. One year after, the International Astronomical Union revisited the definition of a planet and introduced a new category of objects in the Solar System, the "dwarf planets" (objects large enough to be in hydrostatic equilibrium but not large enough to have cleaned their orbit of other minor bodies (see Resolution 5 and $5 \mathrm{~b}$ of the XXVI General Assembly of the IAU for more detail on the definition). With only four icy objects at this moment (the three mentioned above plus Pluto) the exclusive club of the icy dwarf planets $\dagger$ is formed by the TNOs at the higher end of the size distribution and hence we may expect that they exhibit unique characteristics.

$\dagger$ Ceres, in the asteroid belt, is another Dwarf Planet. It is not included in this description as we are focusing on icy objects. 
By virtue of their size and low surface temperatures, these bodies can probably retain most of their original inventory of ices (Schaller \& Brown, 2007; Levy \& Podolak, 2009 ). As a consequence, their visible and near-infrared spectra should show evidences of ices (e.g. nitrogen, methane). Accordingly, the early characterization of the surface composition of these bodies revealed how special they were compared to other TNOs (e.g. Brown et al. 2005b, Licandro et al. 2006a, Licandro et al. 2006b, Pinilla-Alonso et al. 2009, Brown et al. 2007). In addition, these objects are by far the best candidates to have at the moment or occasionally develop on their orbit around the Sun, a bonded atmosphere that exists in equilibrium with the ices on the surface. This atmosphere may or may no collapse on the periods when the object moves further from the Sun, at temperatures such that all the gases condensate onto the surface (for information regarding the detection of an atmosphere around Eris and Makemake read Sicardy et al. 2011; Ortiz et al. 2012; for information on Pluto's atmosphere read Lellouch et al. 2015 and references therein). Moreover, they show some of the highest geometric albedos in the visible, a characteristic that is highly influenced by the physical characteristics of their surface composition i.e. relative abundance of ices vs. silicates or carbonaceous residues, and size of the particles. Also the accrecional and radiogenic heating for these bodies was likely more than sufficient to have caused their internal differentiation (McKinnon et al. 2008).

It is clear that these four giants are peculiar objects what gives the icy dwarf planets an aura of exclusivity. But, are these the only objects in the TNb that, according the IAU definition, can be considered dwarf planets? And if not, which are their physical characteristics? In this paper we review the list of known TNOs and cross it with the most recent estimations of albedo and size to update the list of TNOs candidates to be dwarf planets (CDPs). We also study their main physical characteristics to search for common attributes that can help define other candidates, in the lack of more accurate size estimations.

\section{How many icy dwarf planets are in the Solar System?}

Tancredi \& Favre (2008) review the geophysical criteria to separate a dwarf planet from a regular TNO. They adopt a set of criteria presented as a decision tree. The main idea is to use the data available at that moment to check which minor objects are large enough to overcome the material strength and be in hydrostatic equilibrium. The main parameters affecting the classification are the estimations of the size and shape, and the assumption of a density. Summarizing, their models find a minimum critical size of D $=450 \mathrm{~km}$ for a TNO to be considered a dwarf planet. After applying this criterium to the list of TNOs, they propose a list of 12 very probable and six possible TNOs (always depending on an improvement in the accuracy of the shape or size estimation). They also add that there my be several tens up to more than one hundred objects larger than 450 $\mathrm{km}$ not yet discovered or with no estimation of their size (Tancredi 2009).

In that respect, a recent work (Brown et al. 2015) shows the results of a 7-years survey in search for bright objects covering most of the Northern and Southern hemisphere. The survey probes to be $100 \%$ effective in detecting bright objects $(\mathrm{V} \lesssim 19)$ beyond $25 \mathrm{AU}$, but no new discovery is made. According to this complete study, the remaining probability of finding one or two of this kind of objects in the galactic plane, where the background makes detections more difficult, stays bellow $35 \%$. 


\section{Update of the list of candidates to be dwarf planets}

With no new discoveries, the natural extension of this study is to take advantage of the new estimations of the size and shape of the TNOs and use them to apply the Tancredi \& Favre (2008) criterium $†$. In the last years, the Herschel open time key program "TNOs are Cool!" has improved our knowledge of the physical and thermal properties of a large sample of TNOs (Mullet et al. 2010). When put together with the results of the Spitzer Space Telescope (Stansberry et al. 2008) the improvement in the study of the size of the TNOs is large, not only in the number of estimations (119 objects at this moment) but also in their precision. A compilation of the results of this key program can be accessed via internet at the TNOs Are Cool public database: http://public-tnosarecool.lesia.obspm.fr/Published-results.html. It includes 119 TNOS and centaurs. We use these results to determine which TNOs have a diameter above $450 \mathrm{~km}$. We use a conservative criterium so we will only consider candidates those whose diameter minus the error is above $450 \mathrm{~km}$. The results are listed in table 1.

We find 25 CDPs (including the four already known). We discard three out of the 12 candidates (2002 $\mathrm{TX}_{300}$, Huya and 1996 TL66) in Tancredi \& Favre (2008), and other three $\left(1999 \mathrm{TC}_{36}, 1999 \mathrm{DE}_{9}\right.$ and $\left.2001 \mathrm{QF}_{298}\right)$ out of the six possible dwarf planets. One could not be compared because there are no new estimations.

\subsection{Physical Characteristics and Conclusions}

Here we compare the main physical characteristics (table 1; figure 1) of the CDPs with the sample of TNOs included in the TNOs Are Cool database.

Dynamical classification: The absence of cold classical objects and centaurs in the sample is normal considering that these populations contain small objects (Vilenus et al. 2014; Duffard et al. 2014). The absence of scattered objects could be affected by a bias in the "TNOs Are Cool sample", as that number of scattered objects observed is smaller than for the other dynamical groups.

Albedo: The values of the albedo in the visible range from 0.1 to 0.96 , with a mean value of 0.21 . If we remove from the sample the four known icy dwarf planets, then the maximum is 0.4 and the group of candidates, with a mean value of 0.12 , cannot be distinguish from the rest of the sample of TNOs (figure 1), ranging from 0.1 to 0.35 , with a mean value of 0.12 .

Beaming factor: The values of the beaming factor are also very similar for both groups, with $\eta_{\text {median }}=(1.08,1.11)$ for the TNOs and the CDPs, respectively (figure 1 ). The incidence of large values $(\eta \gtrsim 2)$, indicative of surfaces with high thermal inertia and low values $(\eta \lesssim 1)$, surfaces dominated by roughness effects, is similar among the TNOs and the CDPs.

Binarity: the ratio of binaries detected among the CDPs is $40 \%$. This is double than the ratio of binaries in the rest of the population. However this could be a bias due to the fact that the largest size of the CDPs could produce better data to detect multiple systems (most of them detected from the light curve) making it easier to detract a binary among the CDPs than among the TNOs.

Surface composition: The ratio of clear, tentative and no detections of water ice in the list of CDPs is $(37,21,42) \%$ respectively, very similar to the ratio in the sample of TNOs studied in Barucci et al. (2011; 36, 24, 40 \%) respectively (figure 1). The peculiar surface composition of Eris, Makemake and Pluto has no comparison in the TNb. We can say the same if we consider Haumea and the family of the carbon depleted objects.

$\dagger$ we leave for future consideration the light-curves of the bodies and stick here to the size criterium 

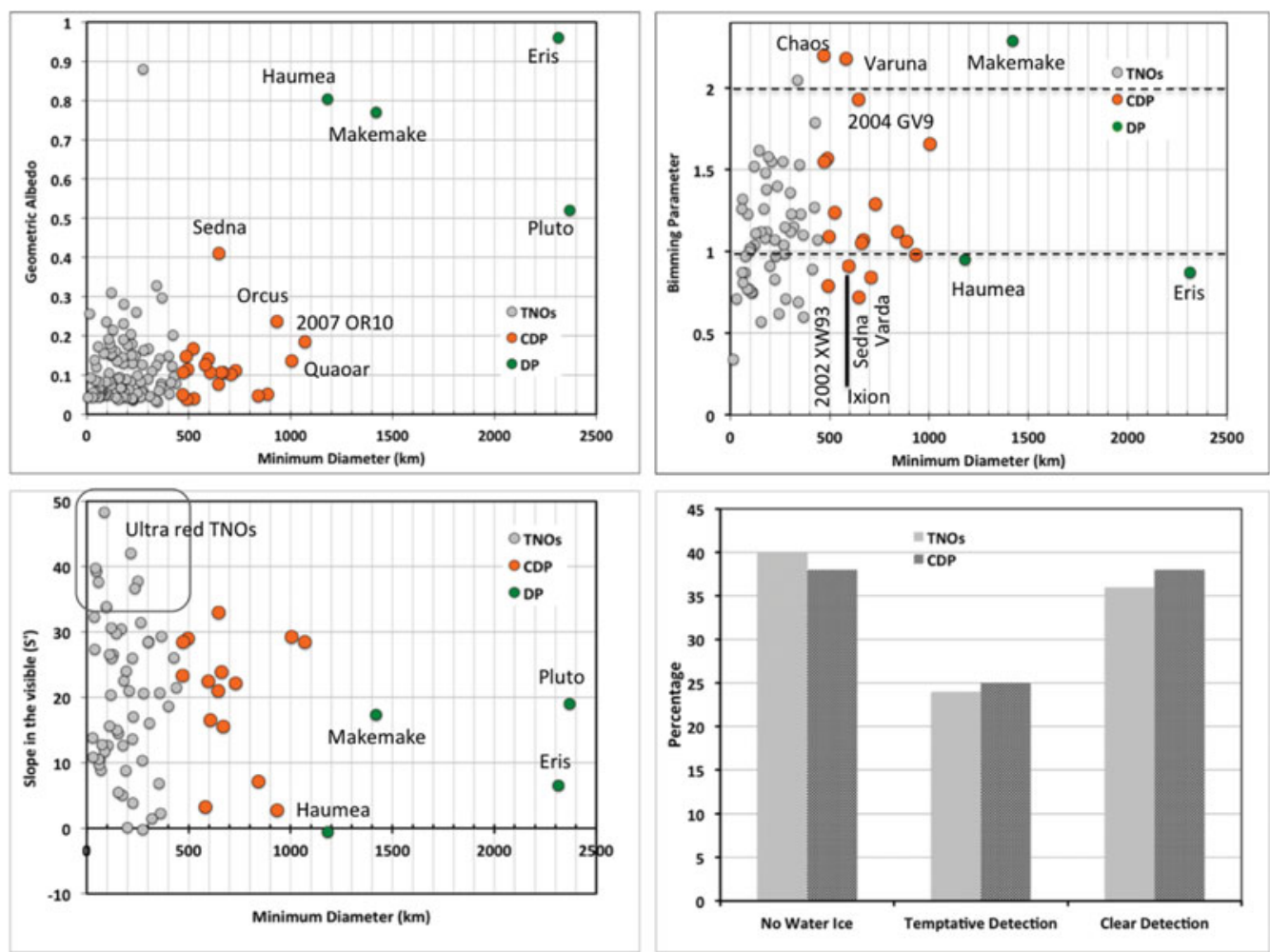

Figure 1. Physical parameters of TNOs, DP and CDPs. See caption of table 1 for references. The distribution of the visible albedo, bumming parameters, color in the visible and detection of water ice for CDP is similar to the distribution for the regular TNOs. None of these parameters could be use to classify a TNO as DP in the absence of a good estimation of size and/or shape. The peculiar charactertics of the DP have no paragon among the TNb.

Neither there is a difference in the slope in the visible of both samples (figure 1). The only possible difference is the absence of extremely red objects among the CDPs, as well as among the DPs, what suggests that the surface of the visible colors of these objects are not dominated by red-dark complex organics.

In conclusion, we have reviewed and compiled here the main physical parameters that can be used to characterize a TNO. We then compare the behavior of two samples, the CDPs (those with $D>450 \mathrm{~km}$ ) and the regular TNOs (all from the list of targets of the TNOs Are Cool program, the ones with a good estimation of the size). We find that, with the exception of the largest ones, the ones classified at the present moment as dwarf planets, there is not a characteristic, other than the size, that is common among the sample. Neither the albedo, or the surface composition, or the dynamical classification, or the thermal properties (beaming factor) help in classifying a regular TNO into the DP class. Only the binarity incidence shows some differences, but we cannot assure that this is not a bias due to the highest quality of the data used in the detection of these multiple systems among the brightest/largest objects.

The actual list of CDPs includes 25 icy objects (see Table 1). It can be refined on the future using the shape information that can be derived from the study of the light-curves. 
Table 1. List of candidate to be dwarf planets and their main physical characteristics

\begin{tabular}{|c|c|c|c|c|c|c|c|c|}
\hline number & name & $\mathbf{D}^{(1)}-\operatorname{err}[\mathrm{km}]$ & $\mathbf{p v} \mathbf{v}_{-e r r}^{(1)+e r r}$ & Dyn. Class ${ }^{(2)}$ & $S^{\prime(3)}$ & {$[\% / 1000 \AA]$} & Water Ice ${ }^{(4)}$ & multiple ${ }^{(5)}$ \\
\hline & Pluto $^{\dagger}$ & 2370 & $0.44-0.59$ & Res & & 12.3 & clear & yes \\
\hline 136199 & Eris & 2314 & $0.96_{-0.04}^{0.04}$ & Det & & 6.5 & no & yes \\
\hline 136472 & Makemake & 1421 & $0.77_{-0.02}^{0.02}$ & Hot & & 17.3 & no & \\
\hline 136108 & Haumea & 1181.7 & $0.80_{-0.1}^{0.06}$ & Hot & & -0.61 & clear & yes \\
\hline 225088 & $2007 \mathrm{OR}_{10}$ & 1070 & $0.19_{-0.05}^{0.08}$ & $\mathrm{SDO}$ & & 28.5 & clear & \\
\hline 50000 & Quaoar & 1005.6 & $0.14_{-0.01}^{0.01}$ & Hot & & 29.2 & clear & yes \\
\hline 90482 & Orcus & 933.6 & $0.24_{0.1}^{0.01}$ & Plut & & 2.8 & clear & yes \\
\hline 307261 & $2002 \mathrm{MS}_{4}$ & 887 & $0.05_{-0.02}^{0.04}$ & Hot & & & & \\
\hline 120347 & Salacia & 842 & $0.05_{0.00}^{0.01}$ & Hot & & 4.1 & & yes \\
\hline 55565 & $2002 \mathrm{AW}_{197}$ & 730 & $0.11_{-0.01}^{0.01}$ & Hot & & 22.1 & no & \\
\hline 174567 & Varda & 708 & $0.10_{0.02}^{0.02}$ & Hot & & & no & yes \\
\hline 55637 & $2002 \mathrm{UX}_{25}$ & 669 & $0.11_{-0.01}^{0.01}$ & Hot & & 15.5 & no & yes \\
\hline 208996 & $2003 \mathrm{AZ}_{84}$ & 660.5 & $0.11_{-0.02}^{0.02}$ & Plut & & 23.9 & clear & yes \\
\hline 90377 & Sedna & 648 & $0.41_{-0.19}^{0.39}$ & $\mathrm{IOO}$ & & 33.0 & clear & \\
\hline 90568 & $2004 \mathrm{GV}_{9}$ & 646 & $0.08_{-0.01}^{0.01}$ & Hot & & 21.0 & no & \\
\hline 145452 & $2005 \mathrm{RN}_{43}$ & 606 & $0.11_{-0.02}^{0.03}$ & Hot & & 16.5 & no & \\
\hline 28978 & Ixion & 597 & $0.14_{-0.01}^{0.01}$ & Plut & & 22.5 & temp & \\
\hline \multirow[t]{2}{*}{20000} & Varuna & 582 & $0.13_{-0.04}^{0.04}$ & Hot & & 3.2 & clear & \\
\hline & $2002 \mathrm{XV}_{93}$ & 526.2 & $0.04_{-0.02}^{0.02}$ & Plut & & & & \\
\hline 229762 & $2007 \mathrm{UK}_{126}$ & 522 & $0.17_{-0.04}^{0.06}$ & Det & & & temp & yes \\
\hline 84522 & $2002 \mathrm{TC}_{302}$ & 496.1 & $0.12_{-0.03}^{0.05}$ & Res & & 29.0 & temp & \\
\hline 78799 & $2002 \mathrm{XW}_{93}$ & 492 & $0.04_{-0.03}^{0.04}$ & Hot & & & & \\
\hline 84922 & $2003 \mathrm{VS}_{2}$ & 488.6 & $0.15_{-0.04}^{0.06}$ & Plut & & & clear & yes \\
\hline 120348 & $2004 \mathrm{TY}_{364}$ & 472 & $0.11_{-0.02}^{0.02}$ & Plut & & 28.4 & temp & \\
\hline 19520 & Chaos & 470 & $0.05_{-0.02}^{0.03}$ & Hot & & 23.3 & no & \\
\hline
\end{tabular}

Notes:

$\dagger$ References for Pluto: size and water ice detection Stern et al. 2015; $S^{\prime}, p_{v}$ Lorenzi et al. (2015);

${ }^{1}$ For the exact reference of the Diameter, and albedo estimation use the TNOs Are Cool public database $^{2}$ : Hot: Hot classical; Plut: plutino; Det: detatched; Res: resonant; IOO: Inner Oort object 3 For the reference of the $S^{\prime}$ or the dynamical class we follow the MBOSS-2 database ${ }^{4}$ We considered no water ice, clear and tentative detections, see Barucci et al. (2011) ${ }^{5}$ For multiple systems use Grundy et al. webpage: http://www2.lowell.edu/users/grundy/tnbs/status.html where a detailed compilation of the refereed publications can be found.

\section{Acknowledgements}

NPA acknowledges funding support from the American Astronomical Society through the AAS InternatIonal travel Grant program. She also acknowledges the SOC of the Focus Meeting 9: Highlights in the Exploration of Small Worlds, for the invitation to participate in this meeting. 


\section{References}

Barucci, M. A., Alvarez-Candal, A. Merlin, F. et al. 2011, Icarus, 214, 297

Brown, R. H., Cruikshank, D. P., \& Pendleton, Y. 1999, ApJ, 519, 101

Brown, M. E., Trujillo, C. A., \& Rabinowitz, D. 2005, IAU Circ., 8577, 1

Brown, M. E., Trujillo, C. A., \& Rabinowitz, D. L. 2005b ApJ, 635, L97

Brown, M. E., Bannister, M. T., \& Schmidt, B. P. 2015, AJ, 149, id. 69

Cruikshank, D. P. 2005, Space Sci. Rev., 116, 421

Duffard, R., Pinilla-Alonso, N., Santos-Sanz, P. et al. 2014, A\&SA, 564, 17

Edgeworth, K. E. 1943, Journal of the British Astronomical Association, 53, 181

Fernandez, J. A. 1980, MNRAS, 192, 481

Jewitt, D. \& Luu, J. 1993, Nature, 362, 730

Jewitt, D., Aussel, H., \& Evans, A 2001, Nature, 411, 446

Kuiper, G. P. 1951, in 50th Anniversary of the Yerkes Observatory and Half a Century of Progress in Astrophysics, ed. J. A. Hynek, 357

Lellouch, E., de Bergh, C., \& Sicardy, B. 2015, Icarus, 246, 268

Leonard, F. C. 1930, Leaflet of the Astronomical Society of the Pacific, 1, 121

Levi, A. \& Podolak, M 2009, Icarus, 202, 681

Licandro, J., Pinilla-Alonso, N., \& Pedani, M. 2006, A\&A, 445, L35

Licandro, J., Grundy, W. M., \& Pinilla-Alonso, N 2006, A\&A, 458, L5

Lorenzi, V., Pinilla-Alonso, N., Licandro, J 2015 A\& A Accepted

McKinnon, W. B., Prialnik, D., Stern, S. A. \& Coradini, A. 2008 The Solar System Beyond Neptune, University of Arizona Press, Tucson, 592, 213

Muller, M. Lellouch, E., et al. 2010, A\& A, 518, L146

Ortiz, J. L., Sicardy, B. Braga-Ribas, F. et al. 2012 Nature, 491, 566

Pinilla-Alonso, N., Brunetto, R., Licandro, J. et al. 2009, A\&A, 496, 547

Santos-Sanz, P., Ortiz, J. L., Aceituno, F. J., Brown, M. E., \& Rabinowitz, D. 2005, IAU Circ., 8577, 2

Schaller, E. \& Brown, M. E. 2007, ApJ, 659, L61

Schmitt, B., De Bergh, C., \& Festou, M. (eds) 1998, Solar System Ices, Astrophysics and Space Science Library, vol 227

Sicardy, B., Ortiz, J. L., Assafin, M. et al. 2011 Nature, 478, 493

Stansberry, J., Grundy, W., Brown, M., et al. 2008 The Solar System Beyond Neptune, University of Arizona Press, Tucson, 592, 161

Stern, S. A., Bagenal, F., \& Ennico, K. 2015, Science, 350, id.aad1815

Tancredi, G. \& Favre, S 2008, Icarus, 195, 851

Tancredi, G 2009 IAU 2009: Icy Bodies in the Solar System, 33

Veillet, C., Parker, J. W., Griffin, I., Marsden, B., Doressoundiram, A., Buie, M., Tholen, D. J., Connelley, M., \& Holman, M. J. 2002, Nature, 416, 711

Vilenius, E., Kiss, C., Muller, T. et al. 2014, A $\mathscr{E} A, 564,18$ 